\title{
General quantum variational calculus
}

\author{
Artur M. C. Brito da Cruz ${ }^{1,2, *}$, Natália Martins ${ }^{2}$ \\ ${ }^{1}$ Escola Superior de Tecnologia de Setúbal, Campus do IPS, Estefanilha, 2914-508 Setúbal, Portugal \\ 2 Center for Research and Development in Mathematics and Applications (CIDMA), \\ Department of Mathematics, University of Aveiro, Portugal
}

\begin{abstract}
We develop a new variational calculus based in the general quantum difference operator recently introduced by Hamza et al. In particular, we obtain optimality conditions for generalized variational problems where the Lagrangian may depend on the endpoints conditions and a real parameter, for the basic and isoperimetric problems, with and without fixed boundary conditions. Our results provide a generalization to previous results obtained for the $q$ - and Hahn-calculus.
\end{abstract}

Keywords General quantum calculus; Hahn's difference operator; Jackson's integral; quantum calculus; calculus of variations; Euler-Lagrange equation; generalized natural boundary conditions; isoperimetric problem.

AMS 2010 subject classifications 39A13, 39A70, 49J05, 49K05, 49K15.

DOI: $10.19139 /$ soic.v6i1.467

\section{Introduction}

It is well-known that many problems in physics, economics, mechanics and calculus of variations can be formulated using integral functionals and that many real life phenomena are characterized by functions that are not differentiable in the classical sense. In the literature there are different approaches to deal with the nondifferentiability of the admissible functions in problems involving integral functionals. In this paper, we are concerned with the quantum approach.

The word quantum is usually referred to the smallest discrete quantity of some physical property and it comes from the Latin word "quantus", which literally means how many. The quantum calculus is usually referred in mathematics as the calculus without limits and it replaces the classical derivative by a difference operator. One type of quantum differential operator is the Hahn quantum derivative introduced in 1949 by Hahn [13]. This operator has been a useful tool in the construction of ortogonal polynomials and in approximation problems [5, 12, 18, 23].

The Hahn calculus has its begining only in 2009 with the Ph.D. thesis of Aldwoah [1], where the author solved an open problem with 60 years: the construction of the inverse operator of Hahn's derivative and the development of the calculus associated with this operator. One year later, the Hahn variational calculus started with the publication of Malinowska and Torres [20]. Due to its importance, new results in Hahn quantum variational calculus have been published in the literature [8, 19]; we also recommend the book [21] for more details on the topic. We refer the interested reader to $[2,3,4,7,10,22]$ for details in calculus of variations based in different quantum operators.

\footnotetext{
${ }^{*}$ Correspondence to: Artur M. C. Brito da Cruz (Email: artur.cruz@estsetubal.ips.pt), Escola Superior de Tecnologia de Setúbal, Campus do IPS, Estefanilha, 2914-508 Setúbal, Portugal.
}

ISSN 2310-5070 (online) ISSN 2311-004X (print)

Copyright (C) 2018 International Academic Press 
Recently, Hamza et al. [14] defined the general quantum difference operator by

$$
D_{\beta}[f](t)=\left\{\begin{array}{cc}
\frac{f(\beta(t))-f(t)}{\beta(t)-t}, & t \neq \beta(t), \\
f^{\prime}(t), & t=\beta(t),
\end{array}\right.
$$

where $\beta: I \rightarrow I$ is a strictly increasing continuous function with a unique fixed point $s_{0} \in I$, where $I$ is a subinterval of $\mathbb{R}$, and such that $\left(t-s_{0}\right)(\beta(t)-t) \leqslant 0$ for all $t \in I$, and developed the general quantum calculus which generalizes the well-known $q$-quantum calculus (also known in the literature as Jackson's calculus) [16] and Hanh's quantum calculus $[9,13]$.

The main purpose of this paper is to study different types of variational problems involving the general quantum difference operator: the basic problem and the isoperimetric problem, with and without fixed boundary conditions, for problems of the calculus of variations with a Lagrangian that may depend on the endpoints conditions and a real parameter.

The structure of the paper is as follows. In Section 2 we present the necessary definitions and results for the general quantum calculus. Our results are given in Section 3: the general quantum Euler-Lagrange equation for the basic problem (Theorem 11), the transversality conditions for the basic problem with free boundary conditions (Theorem 15), necessary optimality conditions for the isoperimetric problem (Theorem 18 and Theorem 19) and a sufficient optimality condition (Theorem 14). To finalize the paper we present some illustrative examples and some concluding remarks.

\section{Preliminaries}

In this section we start with the definition of the general quantum derivative. Let $I \subseteq \mathbb{R}$ be an interval, $f: I \rightarrow \mathbb{R}$ be a real function and $\beta: I \rightarrow I$ be a strictly increasing and continuous function that has a unique fixed point $s_{0} \in I$ that satisfies the following inequality:

$$
\left(t-s_{0}\right)(\beta(t)-t) \leqslant 0 \text { for all } t \in I,
$$

where the equality holds only if $t=s_{0}$. Hamza et al. [14] defined the general quantum difference operator by

$$
D_{\beta}[f](t)=\left\{\begin{array}{cc}
\frac{f(\beta(t))-f(t)}{\beta(t)-t} & , \quad t \neq s_{0} \\
f^{\prime}\left(s_{0}\right) & , \quad t=s_{0}
\end{array}\right.
$$

provided that $f^{\prime}\left(s_{0}\right)$ exists (and is finite) in the usual sense. One says that $f$ is $\beta$-differentiable on $I$ if $f^{\prime}\left(s_{0}\right)$ exists and we call $D_{\beta}[f](t)$ the $\beta$-derivative of $f$ at $t$.

We remark that the general quantum difference operator $D_{\beta}$ can also be defined for strictly increasing and continuous functions $\beta$ that have a unique fixed point $s_{0}$ and satisfy $\left(t-s_{0}\right)(\beta(t)-t) \geqslant 0$, for all $t \in I$ (see [14]).

It is clear that every choice of the function $\beta$ gives a new difference operator and that this operator generalize the well-known Jackson and Hahn operators: if $\beta(t)=q t$, for some $q \in] 0,1[$, then we obtain the Jackson $q$-difference operator [16]; if $\beta(t)=q t+\omega$, for some $q \in] 0,1[$ and $\omega \geqslant 0$, then we obtain the Hahn's quantum difference operator [13]. For a general introduction to the $q$-calculus and the Hahn's calculus, we refer the reader to the book [17] and to the Ph.D. thesis [1], respectively.

In the following, we present the notions and results from the general quantum difference calculus that are needed in this paper. We proceed with some basic properties of the general quantum difference operator.

\section{Theorem 1 ([14])}

Let $f$ and $g$ be $\beta$-differentiable on $I$ and $k \in \mathbb{R}$. One has:

1. $D_{\beta}[f+g](t)=D_{\beta}[f](t)+D_{\beta}[g](t)$; 
2. $D_{\beta}[k f](t)=k D_{\beta}[f](t)$;

3. $D_{\beta}[f g](t)=D_{\beta}[f](t) g(t)+f(\beta(t)) D_{\beta}[g](t)$

$=D_{\beta}[f](t) g(\beta(t))+f(t) D_{\beta}[g](t) ;$

4. $D_{\beta}\left[\frac{f}{g}\right](t)=\frac{D_{\beta}[f](t) g(t)-f(t) D_{\beta}[g](t)}{g(t) g(\beta(t))}$, provided $g(t) g(\beta(t)) \neq 0$

5. $f(\beta(t))=f(t)+(\beta(t)-t) D_{\beta}[f](t)$;

6. If $D_{\beta}[f](t)=0$, for all $t \in I$, then $f(t)=f\left(s_{0}\right), t \in I$.

It is clear that if $f$ is $\beta$-differentiable, then $f$ is continuous at $s_{0}$. We note here that there exist discontinuous functions that are $\beta$-differentiable (for an example see [14]), something that cannot happen in the classical theory and can be seen as an advantage of the general quantum calculus.

In what follows, let us denote

$$
\beta^{k}(t)=\underbrace{\beta \circ \beta \circ \ldots \circ \beta}_{k \text { times }}(t) \quad \text { and } \quad \beta^{0}(t)=t, \quad t \in I .
$$

The following lemma is a useful property of function $\beta$.

Lemma 1 ([14])

The sequence of functions $\left\{\beta^{k}(t)\right\}_{k \in \mathbb{N}_{0}}$ converges uniformly to the fixed point $s_{0}$ on every compact interval $J \subseteq I$ containing $s_{0}$.

Now we present the definition of $\beta$-integral.

Definition 2 ([14])

Let $a, b \in I$ and $a<b$. For $f: I \rightarrow \mathbb{R}$ the $\beta$-integral of $f$ from $a$ to $b$ is given by

$$
\int_{a}^{b} f(t) d_{\beta} t=\int_{s_{0}}^{b} f(t) d_{\beta} t-\int_{s_{0}}^{a} f(t) d_{\beta} t
$$

where

$$
\int_{s_{0}}^{x} f(t) d_{\beta} t=\sum_{k=0}^{+\infty}\left(\beta^{k}(x)-\beta^{k+1}(x)\right) f\left(\beta^{k}(x)\right), x \in I
$$

provided that the series converges at $x=a$ and $x=b . f$ is called $\beta$-integrable on $I$ if the series converge at $a$ and $b$, for all $a, b \in I$.

The following result is also very useful.

Theorem 3 ([14])

If $f \rightarrow \mathbb{R}$ is continuous at $s_{0} \in I$, then $f$ is $\beta$-integrable on $I$.

Remark 1

The $\beta$-integral generalizes the Jackson $q$-integral and the Hahn integral. When $\beta(t)=q t$ for $q \in] 0,1[$, we obtain the Jackson $q$-integral [17]:

$$
\int_{a}^{b} f(t) d_{q} t=\int_{0}^{b} f(t) d_{q} t-\int_{0}^{a} f(t) d_{q} t
$$

where

$$
\int_{0}^{x} f(t) d_{q} t=x(1-q) \sum_{k=0}^{+\infty} q^{k} f\left(x q^{k}\right), x \in I .
$$

When $\beta(t)=q t+\omega$, for $q \in] 0,1[$ and $\omega \geqslant 0$, we obtain the Hahn integral [1]:

$$
\int_{a}^{b} f(t) d_{q, \omega} t=\int_{\omega_{0}}^{b} f(t) d_{q, \omega} t-\int_{\omega_{0}}^{a} f(t) d_{q, \omega} t
$$


where

$$
\int_{\omega_{0}}^{x} f(t) d_{q, \omega} t=(x(1-q)-\omega) \sum_{k=0}^{+\infty} q^{k} f\left(x q^{k}+\omega[k]_{q}\right), x \in I,
$$

and where $\omega_{0}=\frac{\omega}{1-q}$ and $[k]_{q}=\frac{1-q^{k}}{1-q}$.

The $\beta$-integral has the following properties:

Theorem 4 ([14])

Let $f, g: I \rightarrow \mathbb{R}$ be $\beta$-integrable on $I, a, b, c \in I$ and $k \in \mathbb{R}$. Then,

1. $\int_{a}^{b}(f+g)(t) d_{\beta} t=\int_{a}^{b} f(t) d_{\beta} t+\int_{a}^{b} g(t) d_{\beta} t ;$

2. $\int_{a}^{b}(k f)(t) d_{\beta} t=k \int_{a}^{b} f(t) d_{\beta} t$

3. $\int_{a}^{a} f(t) d_{\beta} t=0$;

4. $\int_{a}^{b} f(t) d_{\beta} t=-\int_{b}^{a} f(t) d_{\beta} t$

5. $\int_{a}^{b} f(t) d_{\beta} t=\int_{a}^{c} f(t) d_{\beta} t+\int_{c}^{b} f(t) d_{\beta} t$.

In what follows, for a given $s \in I$, we denote

$$
[s]_{\beta}:=\left\{\beta^{k}(s): k \in \mathbb{N}_{0}\right\} \cup\left\{s_{0}\right\} .
$$

and for $s_{0} \in[a, b] \subseteq I$,

$$
[a, b]_{\beta}:=[a]_{\beta} \cup[b]_{\beta} ;
$$

$[a, b]_{\beta}$ is called the $\beta$-interval with extreme points $a$ and $b$.

Theorem 5 ([14])

Let $f, g: I \rightarrow \mathbb{R}$ be $\beta$-integrable functions on $I$ and let $a, b \in I$ such that $a<s_{0}<b$. If $|f(t)| \leqslant g(t)$ for all $t \in[a, b]_{\beta}$, then
1. $\left|\int_{s_{0}}^{b} f(t) d_{\beta} t\right| \leqslant \int_{s_{0}}^{b} g(t) d_{\beta} t ;$
2. $\left|\int_{s_{0}}^{a} f(t) d_{\beta} t\right| \leqslant-\int_{s_{0}}^{a} g(t) d_{\beta} t ;$
3. $\left|\int_{a}^{b} f(t) d_{\beta} t\right| \leqslant \int_{a}^{b} g(t) d_{\beta} t$.

Consequently, if $g(t) \geqslant 0$, for all $t \in[a, b]_{\beta}$, then the inequalities

$$
\int_{s_{0}}^{b} g(t) d_{\beta} t \geqslant 0 \text { and } \int_{a}^{b} g(t) d_{\beta} t \geqslant 0
$$

hold.

\section{Remark 2}

We remark that:

1. The last result is also true if $a \leqslant s_{0}<b$ or $a<s_{0} \leqslant b$; 
2. It may happens that $g \geqslant 0$ and $\int_{a}^{b} g(t) d_{\beta} t<0$. For example, for the function $g: I \rightarrow \mathbb{R}$ defined by

$$
g(t)=\left\{\begin{array}{cc}
\lambda_{1} & \text { for } \\
0 & \text { otherwise }
\end{array}\right.
$$

for some fixed $\lambda_{1} \in \mathbb{R}^{+}$and $a>s_{0}$, we have

$$
\begin{aligned}
\int_{a}^{b} g(t) d_{\beta} t & =\sum_{k=0}^{+\infty}\left(\beta^{k}(b)-\beta^{k+1}(b)\right) g\left(\beta^{k}(b)\right)-\sum_{k=0}^{+\infty}\left(\beta^{k}(a)-\beta^{k+1}(a)\right) g\left(\beta^{k}(a)\right) \\
& =-\lambda_{1}\left(a-s_{0}\right)<0 .
\end{aligned}
$$

Hamza et al. also have proven the Fundamental Theorem of $\beta$-calculus.

Theorem 6 (Fundamental Theorem of $\beta$-calculus [14])

Let $f: I \rightarrow \mathbb{R}$ be continuous at $s_{0}$. Let $F: I \rightarrow \mathbb{R}$ be the function defined by

$$
F(x)=\int_{s_{0}}^{x} f(t) d_{\beta} t
$$

Then, $F$ is continuous at $s_{0}$. Furthermore, $D_{\beta}[F](x)$ exists for every $x \in I$ and

$$
D_{\beta}[F](x)=f(x) .
$$

Conversely, if $f$ is $\beta$-differentiable on $I$, then

$$
\int_{a}^{b} D_{\beta}[f](t) d_{\beta} t=f(b)-f(a)
$$

for all $a, b \in I$.

Next, we present the $\beta$-integration by parts which is essential to prove our main results.

Theorem 7 ( $\beta$-integration by parts [14])

If $f, g: I \rightarrow \mathbb{R}$ are $\beta$-differentiable and $D_{\beta}[f]$ and $D_{\beta}[g]$ are continuous at $s_{0}$, then

$$
\int_{a}^{b} f(t) D_{\beta}[g](t) d_{\beta} t=\left.f(t) g(t)\right|_{a} ^{b}-\int_{a}^{b} D_{\beta}[f](t) g(\beta(t)) d_{\beta} t, \quad a, b \in I .
$$

The next result is a main tool to obtain necessary optimality conditions for our variational problems.

Lemma 2 (Fundamental lemma of the general quantum variational calculus [14])

Let $f:[a, b]_{\beta} \rightarrow \mathbb{R}$ be continuous at $s_{0}$. Then,

$$
\int_{a}^{b} f(t) \eta(\beta(t)) d_{\beta} t=0
$$

for all functions $\eta:[a, b]_{\beta} \rightarrow \mathbb{R}$ continuous at $s_{0}$ with $\eta(a)=\eta(b)=0$ if and only if $f(t)=0$ for all $t \in[a, b]_{\beta}$.

The following definition and lemma are important for the proofs of our results.

\section{Definition 8}

Let $s \in I$ and $\left.g:[s]_{\beta} \times\right]-\bar{\theta}, \bar{\theta}\left[\rightarrow \mathbb{R}\right.$. We say that $g(t, \cdot)$ is differentiable at $\theta_{0}$ uniformly in $[s]_{\beta}$ if for every $\varepsilon>0$ there exists $\delta>0$ such that

$$
0<\left|\theta-\theta_{0}\right|<\delta \Rightarrow\left|\frac{g(t, \theta)-g\left(t, \theta_{0}\right)}{\theta-\theta_{0}}-\partial_{2} g\left(t, \theta_{0}\right)\right|<\varepsilon
$$

for all $t \in[s]_{\beta}$, where $\partial_{2} g$ denotes the partial derivative of $g$ with respect to the second variable. 
Lemma 3 ([14])

Let $s \in I$. Assume that $\left.g:[s]_{\beta} \times\right]-\bar{\theta}, \bar{\theta}\left[\rightarrow \mathbb{R}\right.$ is differentiable at $\theta_{0}$ uniformly in $[s]_{\beta}$ and that $G(\theta)=$ $\int_{s_{0}}^{s} g(t, \theta) d_{\beta} t$, in a neighborhood of $\theta_{0}$, and $\int_{s_{0}}^{s} \partial_{2} g\left(t, \theta_{0}\right) d_{\beta} t$ exist. Then, $G$ is differentiable at $\theta_{0}$ with

$$
G^{\prime}\left(\theta_{0}\right)=\int_{s_{0}}^{s} \partial_{2} g\left(t, \theta_{0}\right) d_{\beta} t
$$

\section{Main results}

The main objective of this section is to introduce the general quantum variational calculus. For this purpose we consider the following generalized variational problem:

$$
\mathcal{L}[y, \zeta]=\int_{a}^{b} L\left(t, y(\beta(t)), D_{\beta}[y](t), y(a), y(b), \zeta\right) d_{\beta} t \longrightarrow \text { extremize }
$$

where by extremize we mean minimize or maximize, $\zeta$ is a real parameter, and $y \in \mathcal{Y}$, where

$$
\mathcal{Y}:=\left\{y: I \rightarrow \mathbb{R} \mid y \text { and } D_{\beta}[y] \text { are bounded on }[a, b]_{\beta} \text { and continuous at } s_{0}\right\}
$$

endowed with the norm

$$
\|y\|=\sup _{t \in[a, b]_{\beta}}|y(t)|+\sup _{t \in[a, b]_{\beta}}\left|D_{\beta}[y](t)\right| .
$$

We will consider problem (2) with or without fixed boundary conditions and also with an isoperimetric restriction.

The Lagrangian function $L$ is assumed to satisfy the following hypotheses:

(H1) $\left(u_{0}, u_{1}, u_{2}, u_{3}, u_{4}\right) \longrightarrow L\left(t, u_{0}, u_{1}, u_{2}, u_{3}, u_{4}\right)$ is a $C^{1}\left(\mathbb{R}^{5}, \mathbb{R}\right)$ function for any $t \in I$;

$(\mathrm{H} 2) t \longrightarrow L\left(t, y(\beta(t)), D_{\beta}[y](t), y(a), y(b), \zeta\right)$ is continuous at $s_{0}$ for any $y \in \mathcal{Y}$;

(H3) functions $t \rightarrow \partial_{i+2} L\left(t, y(\beta(t)), D_{\beta}[y](t), y(a), y(b), \zeta\right), i=0,1, \ldots, 4$ belong to $\mathcal{Y}$, for all $y \in \mathcal{Y}$;

where $\partial_{i} L$ denotes the partial derivative of $L$ with respect to its $i$ th-coordinate.

The motivation to study generalized variational problems where the Lagrangian depends on the state values can be found in economics problems ([24]); the dependence of a real parameter is important, for example, in physical problems ([15]).

In Section 3.1 we derive optimality conditions for problem (2) in the class of functions $y \in \mathcal{Y}$ satisfying the boundary conditions:

$$
y(a)=\alpha \text { and } y(b)=\gamma
$$

for some fixed $\alpha, \gamma \in \mathbb{R}$. Then we consider problem (2) without fixed boundary conditions. In this case, two additional necessary conditions, usually called natural boundary conditions or transversality conditions, are obtained. Finally, in Section 3.2 we study problem (2) in the class of functions $y \in \mathcal{Y}$ satisfying the integral constraint

$$
\mathcal{I}[y, \zeta]=\int_{a}^{b} F\left(t, y(\beta(t)), D_{\beta}[y](t), y(a), y(b), \zeta\right) d_{\beta} t=\tau
$$

for some $\tau \in \mathbb{R}$.

\subsection{Generalized quantum variational problems}

We begin this section with some basic definitions that are useful in what follows.

Definition 9

We say that $y$ is an admissible function for problem (2) if $y \in \mathcal{Y}$; $y \in \mathcal{Y}$ is said an admissible function for problem (2)-(3) if $y$ satisfies the boundary conditions (3). We say that a function $\eta \in \mathcal{Y}$ is an admissibile variation for problem (2)-(3) if $\eta(a)=\eta(b)=0$. 


\section{Definition 10}

We say that $\left(y_{*}, \zeta_{*}\right)$ is a local minimimizer (resp. local maximizer) for problem (2) ((and (2)-(3)) if $y_{*}$ is an admissible function and there exists $\gamma>0$ such that

$$
\mathcal{L}\left[y_{*}, \zeta_{*}\right] \leqslant \mathcal{L}[y, \zeta] \quad\left(\text { resp. } \quad \mathcal{L}\left[y_{*}, \zeta_{*}\right] \geqslant \mathcal{L}[y, \zeta]\right)
$$

for admissible function $y$ and $\zeta \in \mathbb{R}$ with $\left\|y_{*}-y\right\|<\gamma$ and $\left|\zeta-\zeta_{*}\right|<\gamma$.

In what follows and in order to simplify expressions, we introduce the notation

$$
[y ; \zeta]_{\beta}(t):=\left(t, y(\beta(t)), D_{\beta}[y](t), y(a), y(b), \zeta\right) .
$$

Therefore,

$$
\mathcal{L}[y, \zeta]=\int_{a}^{b} L[y ; \zeta]_{\beta}(t) d_{\beta} t .
$$

For an admissible variation $\eta$, an admissible function $y$, a real parameter $\zeta$, and a real $\delta$, we define the function $\phi:]-\bar{\epsilon}, \bar{\epsilon}[\rightarrow \mathbb{R}$ by

$$
\phi(\epsilon)=\mathcal{L}[y+\epsilon \eta, \zeta+\epsilon \delta] .
$$

The following result is an immediate consequence of Lemma 3.

\section{Lemma 4}

For an admissible variation $\eta$, an admissible function $y$, and $\delta \in \mathbb{R}$, let

$$
g(t, \epsilon)=L[y+\epsilon \eta ; \zeta+\epsilon \delta]_{\beta}(t)
$$

for $\epsilon \in]-\bar{\epsilon}, \bar{\epsilon}[$. Assume that:

(i) $g(t, \cdot)$ is differentiable at 0 uniformly in $[a, b]_{\beta}$;

(ii) $\int_{s_{0}}^{a} L[y+\epsilon \eta ; \zeta+\epsilon \delta]_{\beta}(t) d_{\beta} t$ and $\int_{s_{0}}^{b} L[y+\epsilon \eta ; \zeta+\epsilon \delta]_{\beta}(t) d_{\beta} t$ exist for $\epsilon$ in a neighborhood of 0 ;

(iii) $\int_{s_{0}}^{a} \partial_{2} g(t, 0) d_{\beta} t$ and $\int_{s_{0}}^{b} \partial_{2} g(t, 0) d_{\beta} t$ exist.

Then,

$$
\begin{aligned}
& \phi^{\prime}(0)=\int_{a}^{b}\left(\partial_{2} L[y ; \zeta]_{\beta}(t) \cdot \eta(\beta(t))+\partial_{3} L[y ; \zeta]_{\beta}(t) \cdot D_{\beta}[\eta](t)\right. \\
& \left.+\partial_{4} L[y ; \zeta]_{\beta}(t) \cdot \eta(a)+\partial_{5} L[y ; \zeta]_{\beta}(t) \cdot \eta(b)+\partial_{6} L[y ; \zeta]_{\beta} \cdot \delta\right) d_{\beta} t
\end{aligned}
$$

We can now prove a necessary condition for an admissible pair $\left(y_{*}, \zeta_{*}\right)$ to be a local extremizer of problem (2)-(3).

Theorem 11 (General quantum Euler-Lagrange equation)

Under hypotheses (H1)-(H3) and (i)-(iii) of Lemma 4 on the Lagrangian function $L$, if the pair $\left(y_{*}, \zeta_{*}\right)$ is a local extremizer for problem (2)-(3), then $\left(y_{*}, \zeta_{*}\right)$ satisfies the $\beta$-Euler-Lagrange equation

$$
D_{\beta}\left[\partial_{3} L\right][y ; \zeta]_{\beta}(t)=\partial_{2} L[y ; \zeta]_{\beta}(t), \quad t \in[a, b]_{\beta}
$$

and

$$
\int_{a}^{b} \partial_{6} L[y ; \zeta]_{\beta}(t) d_{\beta} t=0 .
$$


Proof

Let $\left(y_{*}, \zeta_{*}\right)$ be a local extremizer for problem (2)-(3), $\eta$ an admissible variation and $\delta \in \mathbb{R}$. A necessary condition for $\left(y_{*}, \zeta_{*}\right)$ to be an extremizer is given by $\phi^{\prime}(0)=0$. Therefore, by Lemma 4 ,

$$
\begin{array}{r}
\int_{a}^{b}\left(\partial_{2} L\left[y_{*} ; \zeta_{*}\right]_{\beta}(t) \cdot \eta(\beta(t))+\partial_{3} L\left[y_{*} ; \zeta_{*}\right]_{\beta}(t) \cdot D_{\beta}[\eta](t)\right. \\
\left.+\partial_{4} L\left[y_{*} ; \zeta_{*}\right]_{\beta}(t) \cdot \eta(a)+\partial_{5} L\left[y_{*} ; \zeta_{*}\right]_{\beta}(t) \cdot \eta(b)+\partial_{6} L\left[y_{*} ; \zeta_{*}\right]_{\beta} \cdot \delta\right) d_{\beta} t=0 .
\end{array}
$$

Using $\beta$-integration by parts and observing that $\eta(a)=\eta(b)=0$, we obtain

$$
\begin{aligned}
\int_{a}^{b} \partial_{3} L\left[y_{*} ; \zeta_{*}\right]_{\beta}(t) \cdot D_{\beta}[\eta](t) d_{\beta} t & =\left.\partial_{3} L\left[y_{*} ; \zeta_{*}\right]_{\beta}(t) \cdot \eta(t)\right|_{a} ^{b}-\int_{a}^{b} D_{\beta}\left[\partial_{3} L\right]\left[y_{*} ; \zeta_{*}\right]_{\beta}(t) \cdot \eta(\beta(t)) d_{\beta} t \\
& =-\int_{a}^{b} D_{\beta}\left[\partial_{3} L\right]\left[y_{*} ; \zeta_{*}\right]_{\beta}(t) \cdot \eta(\beta(t)) d_{\beta} t
\end{aligned}
$$

and therefore

$$
\int_{a}^{b}\left(\partial_{2} L\left[y_{*} ; \zeta_{*}\right]_{\beta}(t)-D_{\beta}\left[\partial_{3} L\right]\left[y_{*} ; \zeta_{*}\right]_{\beta}(t)\right) \eta(\beta(t)) d_{\beta} t+\int_{a}^{b} \partial_{6} L\left[y_{*} ; \zeta_{*}\right]_{\beta}(t) \cdot \delta d_{\beta} t=0 .
$$

Taking $\delta=0$ and using the arbitrariness of $\eta$, we can conclude by Lemma 2, that

$$
\partial_{2} L\left[y_{*} ; \zeta_{*}\right]_{\beta}(t)-D_{\beta}\left[\partial_{3} L\right]\left[y_{*} ; \zeta_{*}\right]_{\beta}(t)=0,
$$

for all $t \in[a, b]_{\beta}$. Taking the admissible variation to be null we obtain

$$
\int_{a}^{b} \partial_{6} L\left[y_{*} ; \zeta_{*}\right]_{\beta}(t) \cdot \delta d_{\beta} t=0
$$

and, by the arbitrariness of $\delta$, we get

$$
\int_{a}^{b} \partial_{6} L\left[y_{*} ; \zeta_{*}\right]_{\beta}(t) d_{\beta} t=0
$$

as intended.

In the particular case where the Lagrangian function $L$ does not depend on the state values $y(a)$ and $y(b)$, and on the real parameter $\zeta$, we obtain the following necessary optimality condition for the simplest variational problem, where the Lagrangian $L$ satisfies conditions (H1)-(H3) and (i)-(iii) of Lemma 4, adapted to the case where $L$ depends only on the arguments $\left(t, y(\beta(t)), D_{\beta}[y](t)\right)$.

Corollary 1

If $y_{*}$ is a local extremizer to problem

$$
\mathcal{L}[y]=\int_{a}^{b} L\left(t, y(\beta(t)), D_{\beta}[y](t)\right) d_{\beta} t \longrightarrow \text { extremize }
$$

satisfying the boundary conditions (3), then $y_{*}$ satisfies the Euler-Lagrange equation

$$
D_{\beta}\left[\partial_{3} L\right]\left(t, y(\beta(t)), D_{\beta}[y](t)\right)=\partial_{2} L\left(t, y(\beta(t)), D_{\beta}[y](t)\right)
$$

for all $t \in[a, b]_{\beta}$.

Remark 3

If we specify the function $\beta$ and consider the simplest problem of calculus of variations, we obtain the following necessary optimality conditions, proven in [6] and [20], respectively: 
1. if $\beta(t)=q t$, for $q \in] 0,1$, then we obtain the $q$-Euler-Lagrange equation

$$
\left.\partial_{2} L\left(t, y(q t), D_{q}[y](t)\right)=D_{q}\left[\partial_{3} L\right](t, y(q t)), D_{q}[y](t)\right) ;
$$

2. if $\beta(t)=q t+\omega$, for $q \in] 0,1[$ and $\omega \geqslant 0$, then we obtain the Hahn's quantum Euler-Lagrange equation

$$
\partial_{2} L\left(t, y(q t+\omega), D_{q, \omega}[y](t)\right)=D_{q, \omega}\left[\partial_{3} L\right]\left(t, y(q t+\omega), D_{q, \omega}[y](t)\right) .
$$

\section{Definition 12}

We say that a pair $(y, \zeta)$ is an extremal to problem (2)-(3) if $(y, \zeta)$ satisfies the $\beta$-Euler-Lagrange equation (5) and condition (6).

In order to prove a sufficient optimality condition for problem (2)-(3), that is useful in the search of extremizers, we introduce the following definition.

Definition 13

Given a function $L: I \times \mathbb{R}^{5} \rightarrow \mathbb{R}$, we say that $L\left(t, u_{0}, \ldots, u_{4}\right)$ is jointly convex (resp. jointly concave) in $\left(u_{0}, \ldots, u_{4}\right)$ if and only if $\partial_{i} L, i=2, \ldots, 6$, are continuous and satisfy the following condition:

$$
L\left(t, u_{0}+\bar{u}_{0}, \ldots, u_{4}+\bar{u}_{4}\right)-L\left(t, u_{0}, \ldots, u_{4}\right) \underset{\text { (resp. } \leqslant)}{\geqslant} \sum_{i=2}^{6} \partial_{i} L\left(t, u_{0}, \ldots, u_{4}\right) \bar{u}_{i-2}
$$

for all $\left(t, u_{0}, \ldots, u_{4}\right),\left(t, u_{0}+\bar{u}_{0}, \ldots, u_{4}+\bar{u}_{4}\right) \in[a, b]_{\beta} \times \mathbb{R}^{5}$.

Theorem 14 (Sufficient optimality condition)

Suppose that $a \leqslant s_{0}<b$ or $a<s_{0} \leqslant b$ and let $L\left(t, u_{0}, \ldots, u_{4}\right)$ be jointly convex (resp. jointly concave) in $\left(u_{0}, \ldots, u_{4}\right)$. If $\left(y_{*}, \zeta_{*}\right)$ is an extremal, then $\left(y_{*}, \zeta_{*}\right)$ is a global minimizer (resp. global maximizer) to problem (2)-(3).

Proof

Without loss of generality, let us consider that $L$ is jointly convex in $\left(u_{0}, \ldots, u_{4}\right)$. For any admissible variation $\eta$ and $\delta \in \mathbb{R}$, it follows that

$$
\begin{aligned}
& \mathcal{L}\left[y_{*}+\eta, \zeta_{*}+\delta\right]-\mathcal{L}\left[y_{*}, \zeta_{*}\right] \\
& =\int_{a}^{b}\left(L\left[y_{*}+\eta ; \zeta_{*}+\delta\right]_{\beta}(t)-L\left[y_{*} ; \zeta_{*}\right]_{\beta}(t)\right) d_{\beta} t \\
& \geqslant \int_{a}^{b}\left(\partial_{2} L\left[y_{*} ; \zeta_{*}\right]_{\beta}(t) \cdot \eta(\beta(t))+\partial_{3} L\left[y_{*} ; \zeta_{*}\right]_{\beta}(t) \cdot D_{\beta}[\eta](t)\right. \\
& \left.+\partial_{4} L\left[y_{*} ; \zeta_{*}\right]_{\beta}(t) \cdot \eta(a)+\partial_{5} L\left[y_{*} ; \zeta_{*}\right]_{\beta}(t) \cdot \eta(b)+\partial_{6} L\left[y_{*} ; \zeta_{*}\right]_{\beta}(t) \cdot \delta\right) d_{\beta} t .
\end{aligned}
$$

Using integration by parts, the fact that $\eta(a)=\eta(b)=0$ and observing that $\left(y_{*}, \zeta_{*}\right)$ satisfies the $\beta$-Euler-Lagrange equation and condition (6), we get

$$
\mathcal{L}\left[y_{*}, \zeta_{*}\right] \leqslant \mathcal{L}\left[y_{*}+\eta, \zeta_{*}+\delta\right]
$$

as intended.

\section{Remark 4}

We emphasize that condition $a \leqslant s_{0}<b$ or $a<s_{0} \leqslant b$ is mandatory in Theorem 14 in order to apply Theorem 5 .

Another important question is to find optimality conditions that extremals has to satisfy in the case we have no boundary constraints on the set of admissible functions. 
Theorem 15 (Natural boundary conditions)

Under hypotheses (H1)-(H3) and (i)-(iii) of Lemma 4 on the Lagrangian function $L$, if the pair $\left(y_{*}, \zeta_{*}\right)$ is a local extremizer for problem (2), then $\left(y_{*}, \zeta_{*}\right)$ satisfies the $\beta$-Euler-Lagrange equation (5) and condition (6). Moreover,

1. if $y(a)$ is free, then the natural boundary condition

$$
\partial_{3} L\left[y_{*} ; \zeta_{*}\right]_{\beta}(a)=\int_{a}^{b} \partial_{4} L\left[y_{*} ; \zeta_{*}\right]_{\beta}(t) d_{\beta} t
$$

holds;

2. if $y(b)$ is free, then the natural boundary condition

$$
\partial_{3} L\left[y_{*} ; \zeta_{*}\right]_{\beta}(b)=-\int_{a}^{b} \partial_{5} L\left[y_{*} ; \zeta_{*}\right]_{\beta}(t) d_{\beta} t
$$

holds.

Proof

Let $\eta \in \mathcal{Y}$ and $\delta \in \mathbb{R}$ be arbitrary. A necessary condition for $\left(y_{*}, \zeta_{*}\right)$ to be an extremizer is given by

$$
\phi^{\prime}(0)=0
$$

where $\phi(\epsilon)=\mathcal{L}\left[y_{*}+\epsilon \eta, \zeta_{*}+\epsilon \delta\right]$. By Lemma 4, we conclude that

$$
\begin{aligned}
& \int_{a}^{b}\left(\partial_{2} L\left[y_{*} ; \zeta_{*}\right]_{\beta}(t) \cdot \eta(\beta(t))+\partial_{3} L\left[y_{*} ; \zeta_{*}\right]_{\beta}(t) \cdot D_{\beta}[\eta](t)\right. \\
& \left.+\partial_{4} L\left[y_{*} ; \zeta_{*}\right]_{\beta}(t) \cdot \eta(a)+\partial_{5} L\left[y_{*} ; \zeta_{*}\right]_{\beta}(t) \cdot \eta(b)+\partial_{6} L\left[y_{*} ; \zeta_{*}\right]_{\beta}(t) \cdot \delta\right) d_{\beta} t=0
\end{aligned}
$$

and using integration by parts we obtain

$$
\begin{aligned}
& \left.\partial_{3} L\left[y_{*} ; \zeta_{*}\right]_{\beta}(t) \cdot \eta(t)\right|_{a} ^{b}+\int_{a}^{b}\left(\partial_{2} L\left[y_{*} ; \zeta_{*}\right]_{\beta}(t)-D_{\beta}\left[\partial_{3} L\right]\left[y_{*} ; \zeta_{*}\right]_{\beta}(t)\right) d_{\beta} t \\
& +\int_{a}^{b}\left(\partial_{4} L\left[y_{*} ; \zeta_{*}\right]_{\beta}(t) \cdot \eta(a)+\partial_{5} L\left[y_{*} ; \zeta_{*}\right]_{\beta}(t) \cdot \eta(b)\right) d_{\beta} t \\
& +\int_{a}^{b} \partial_{6} L\left[y_{*} ; \zeta_{*}\right]_{\beta}(t) \cdot \delta d_{\beta} t=0 .
\end{aligned}
$$

Since no boundary conditions are imposed, $\eta$ do not need to vanish at the endpoints. However, this last equation must be satisfied for all $\eta \in \mathcal{Y}$ and in particular for the functions that do vanish at the endpoints. Using the same arguments used in the proof of Theorem 5 we conclude that $\left(y_{*}, \zeta_{*}\right)$ satisfies the general Euler-Lagrange equation

$$
\partial_{2} L\left[y_{*} ; \zeta_{*}\right]_{\beta}(t)=D_{\beta}\left[\partial_{3} L\right]\left[y_{*} ; \zeta_{*}\right]_{\beta}(t), \quad t \in[a, b]_{\beta}
$$

and condition $\int_{a}^{b} \partial_{6} L\left[y_{*} ; \zeta_{*}\right]_{\beta}(t) d_{\beta} t=0$.

1. Suppose that $y(a)$ is free. If $y(b)$ is fixed, then $\eta(b)=0$; if $y(b)$ is free then we can restrict to all functions $\eta \in \mathcal{Y}$ such that $\eta(b)=0$. In this case, using general quantum Euler-Lagrange equation and taking $\delta=0$, we conclude that

$$
\partial_{3} L\left[y_{*} ; \zeta_{*}\right]_{\beta}(a) \cdot \eta(a)=\int_{a}^{b} \partial_{4} L\left[y_{*} ; \zeta_{*}\right]_{\beta}(t) \cdot \eta(a) d_{\beta} t .
$$

From the arbitrariness of $\eta$ it follows that

$$
\partial_{3} L\left[y_{*} ; \zeta_{*}\right]_{\beta}(a)=\int_{a}^{b} \partial_{4} L\left[y_{*} ; \zeta_{*}\right]_{\beta}(t) d_{\beta} t .
$$


2. Suppose that $y(b)$ is free. If $y(a)$ is fixed, then $\eta(a)=0$; if $y(a)$ is free then we can restrict to all functions $\eta \in \mathcal{Y}$ such that $\eta(a)=0$. In this case, using general quantum Euler-Lagrange equation and choosing $\delta=0$, it follows that $\partial_{3} L\left[y_{*} ; \zeta_{*}\right]_{\beta}(b)=-\int_{a}^{b} \partial_{5} L\left[y_{*} ; \zeta_{*}\right]_{\beta}(t) d_{\beta} t$.

The proof is complete.

For the special case where $\beta(t)=q t+\omega$, for some $q \in] 0,1[$ and $\omega \geqslant 0$, and the Lagrangian $L$ does not depend on the real parameter $\zeta$, we obtain the following result of the Hahn variational calculus, where we suppose that $L$ satisfies conditions (H1)-(H3) and (i)-(iii) of Lemma 4 adapted for the case that $L$ depends only on the arguments $\left(t, y(q t+\omega), D_{q, \omega}[y](t), y(a), y(b)\right)$. In order to simplify the expressions, we denote

$$
[y]_{q, \omega}(t):=\left(t, y(q t+\omega), D_{q, \omega}[y](t), y(a), y(b)\right)
$$

and

$$
[a, b]_{q, \omega}:=\left\{q^{n} a+\omega[n]_{q}\right\} \cup\left\{q^{n} b+\omega[n]_{q}\right\} \cup\left\{\omega_{0}\right\}
$$

(see Remark 1 for more details).

Corollary 2 ([19])

If $y_{*}$ is a local extremizer for problem (2), then $y_{*}$ satisfies the quantum Euler-Lagrange equation

$$
\partial_{2} L[y]_{q, \omega}(t)=D_{q, \omega}\left[\partial_{3} L\right][y]_{q, \omega}(t), \quad t \in[a, b]_{q, \omega} .
$$

Moreover,

1. if $y(a)$ is free, then the natural boundary condition

$$
\partial_{3} L[y]_{q, \omega}(a)=\int_{a}^{b} \partial_{4} L[y]_{q, \omega}(t) d_{q, \omega} t
$$

holds;

2. if $y(b)$ is free, then the natural boundary condition

$$
\partial_{3} L[y]_{q, \omega}(b)=-\int_{a}^{b} \partial_{5} L[y]_{q, \omega}(t) d_{q, \omega} t
$$

holds.

In the particular case where the Lagrangian function $L$ does not depend on the state values $y(a)$ and $y(b)$, and on the real parameter $\zeta$, and under appropriate assumptions on the Lagrangian, we obtain the following necessary optimality conditions.

Corollary 3

If $y_{*}$ is a local extremizer to problem

$$
\mathcal{L}[y]=\int_{a}^{b} L\left(t, y(\beta(t)), D_{\beta}[y](t)\right) d_{\beta} t \longrightarrow \text { extremize },
$$

then $y_{*}$ satisfies the Euler-Lagrange equation

$$
D_{\beta}\left[\partial_{3} L\right]\left(t, y(\beta(t)), D_{\beta}[y](t)\right)=\partial_{2} L\left(t, y(\beta(t)), D_{\beta}[y](t)\right)
$$

for all $t \in[a, b]_{\beta}$ and 
1. if $y(a)$ is free, then the natural boundary condition

$$
\partial_{3} L\left(t, y(\beta(t)), D_{\beta}[y](t)\right)(a)=0
$$

holds;

2. if $y(b)$ is free, then the natural boundary condition

$$
\partial_{3} L\left(t, y(\beta(t)), D_{\beta}[y](t)\right)(b)=0
$$

holds.

\section{Remark 5}

Note that the sufficient condition for optimality presented in Theorem 14 is still valid for variational problems with free boundary conditions.

\subsection{Generalized quantum isoperimetric problems}

In this section we consider problem (2) when we are in presence of an integral constraint. This kind of variational problems is know in the literature as isoperimetric problems. The problem that consist in the search of a closed plane curve of a given perimeter which encloses the greatest area is a well-known example of an isoperimetric problem.

The quantum isoperimetric problem that we consider here consists in extremizing the functional

$$
\mathcal{L}[y, \zeta]=\int_{a}^{b} L\left(t, y(\beta(t)), D_{\beta}[y](t), y(a), y(b), \zeta\right) d_{\beta} t
$$

in the class of functions $y \in \mathcal{Y}$ satisfying the boundary conditions (3) and the integral constraint

$$
\mathcal{I}[y, \zeta]=\int_{a}^{b} F\left(t, y(\beta(t)), D_{\beta}[y](t), y(a), y(b), \zeta\right) d_{\beta} t=\tau
$$

for some fixed value $\tau \in \mathbb{R}$.

Before presenting necessary optimality conditions for such kind of variational problems, we first give some basic definitions.

\section{Definition 16}

We say that a pair $\left(y_{*}, \zeta_{*}\right)$ is a local minimizer (resp. local maximizer) to the isoperimetric problem (8)-(9) if there exists $\gamma>0$ such that $\mathcal{L}\left[y_{*}, \zeta_{*}\right] \leqslant \mathcal{L}[y, \zeta]$ (resp. $\mathcal{L}\left[y_{*}, \zeta_{*}\right] \geqslant \mathcal{L}[y, \zeta]$ ) for all functions $y \in \mathcal{Y}$ and all $\zeta \in \mathbb{R}$ satisfying the integral constraint (9) with $\left\|y_{*}-y\right\|<\gamma$ and $\left|\zeta_{*}-\zeta\right|<\gamma$.

\section{Definition 17}

We say that $(y, \zeta)$ is a normal extremizer to the isoperimetric problem (8)-(9) if $(y, \zeta)$ is a local extremizer to problem (8)-(9) that is not an extremal to functional $\mathcal{I}$; if $(y, \zeta)$ is a local extremizer and an extremal to functional $\mathcal{I}$ we say that $(y, \zeta)$ is an abnormal extremizer.

Now we are ready to prove necessary optimality conditions for isoperimetric problems, with and without fixed boundary conditions, for the particular case of normal extremizers.

Theorem 18 (Necessary optimality conditions for normal extremizers to isoperimetric problems)

Let us suppose that $L$ and $F$ satisfy hypotheses (H1)-(H3) and (i)-(iii) of Lemma 4 . If $\left(y_{*}, \zeta_{*}\right)$ is a normal extremizer to the isoperimetric problem (8)-(9), then there exists a real number $\lambda \in \mathbb{R}$ such that $\left(y_{*}, \zeta_{*}\right)$ satisfies the following conditions

and

$$
\partial_{2} H[y ; \zeta]_{\beta}(t)=D_{\beta}\left[\partial_{3} H\right][y ; \zeta]_{\beta}(t), \quad t \in[a, b]_{\beta}
$$

$$
\int_{a}^{b} \partial_{6} H[y ; \zeta]_{\beta}(t) d_{\beta} t=0
$$

where $H=L-\lambda F$. Moreover, 
1. if $y(a)$ is free, then

$$
\partial_{3} H\left[y_{*} ; \zeta_{*}\right]_{\beta}(a)=\int_{a}^{b} \partial_{4} H\left[y_{*} ; \zeta_{*}\right]_{\beta}(t) d_{\beta} t
$$

2. if $y(b)$ is free, then

$$
\partial_{3} H\left[y_{*} ; \zeta_{*}\right]_{\beta}(b)=-\int_{a}^{b} \partial_{5} H\left[y_{*} ; \zeta_{*}\right]_{\beta}(t) d_{\beta} t
$$

Proof

Let us suppose that $\left(y_{*}, \zeta_{*}\right)$ is a normal extremizer for the isoperimetric problem (8)-(9). Let us define two real functions $\phi, \psi: \mathbb{R}^{2} \rightarrow \mathbb{R}$ by

$$
\begin{aligned}
\phi\left(\varepsilon_{1}, \varepsilon_{2}\right) & =\mathcal{L}\left[y_{*}+\varepsilon_{1} \eta_{1}+\varepsilon_{2} \eta_{2}, \zeta_{*}+\varepsilon_{1} \delta_{1}+\varepsilon_{2} \delta_{2}\right] \\
\psi\left(\varepsilon_{1}, \varepsilon_{2}\right) & =\mathcal{I}\left[y_{*}+\varepsilon_{1} \eta_{1}+\varepsilon_{2} \eta_{2}, \zeta_{*}+\varepsilon_{1} \delta_{1}+\varepsilon_{2} \delta_{2}\right]-\tau,
\end{aligned}
$$

where $\eta_{1}$ is an arbitrary variation, $\eta_{2}$ is a fixed variation (that we will choose later), $\delta_{1}$ is an arbitrary real number and $\delta_{2}$ is a fixed real number (that we will choose later).

Note that

$$
\begin{aligned}
\partial_{2} \psi(0,0)= & \int_{a}^{b}\left(\partial_{2} F\left[y_{*} ; \zeta_{*}\right](t) \cdot \eta_{2}(\beta(t))+\partial_{3} F\left[y_{*} ; \zeta_{*}\right](t) \cdot D_{\beta}\left[\eta_{2}\right](t)\right. \\
& +\partial_{4} F\left[y_{*}, \zeta_{*}\right](t) \cdot \eta_{2}(a)+\partial_{5} F\left[y_{*} ; \zeta_{*}\right](t) \cdot \eta_{2}(b) \\
& \left.+\partial_{6} F\left[y_{*} ; \zeta_{*}\right](t) \cdot \delta_{2}\right) d_{\beta} t
\end{aligned}
$$

Using integration by parts formula we get that

$$
\begin{aligned}
\partial_{2} \psi(0,0) & =\int_{a}^{b}\left(\partial_{2} F\left[y_{*} ; \zeta_{*}\right](t)-D_{\beta}\left[\partial_{3} F\right]\left[y_{*} ; \zeta_{*}\right]_{\beta}(t)\right) \cdot \eta_{2}(\beta(t)) d_{\beta} t \\
& +\int_{a}^{b}\left(\partial_{4} F\left[y_{*} ; \zeta_{*}\right](t) \cdot \eta_{2}(a)+\partial_{5} F\left[y_{*} ; \zeta_{*}\right](t) \cdot \eta_{2}(b)+\partial_{6} F\left[y_{*} ; \zeta_{*}\right](t) \cdot \delta_{2}\right) d_{\beta} t \\
& +\partial_{3} F\left[y_{*} ; \zeta_{*}\right](t) \cdot \eta_{2}(b)-\partial_{3} F\left[y_{*} ; \zeta_{*}\right](t) \cdot \eta_{2}(a) .
\end{aligned}
$$

Since $\left(y_{*}, \zeta_{*}\right)$ is not an extremal of $\mathcal{I}$, then it can happen three cases:

(i) $\partial_{2} F\left[y_{*} ; \zeta_{*}\right]_{\beta}(t) \neq D_{\beta}\left[\partial_{3} F\right]\left[y_{*} ; \zeta_{*}\right]_{\beta}(t)$ and $\int_{a}^{b} \partial_{6} F\left[y_{*} ; \zeta_{*}\right]_{\beta}(t) d_{\beta} t=0$;

(ii) $\partial_{2} F\left[y_{*} ; \zeta_{*}\right]_{\beta}(t)=D_{\beta}\left[\partial_{3} F\right]\left[y_{*} ; \zeta_{*}\right]_{\beta}(t)$ and $\int_{a}^{b} \partial_{6} F\left[y_{*} ; \zeta_{*}\right]_{\beta}(t) d_{\beta} t \neq 0$;

(iii) $\partial_{2} F\left[y_{*} ; \zeta_{*}\right]_{\beta}(t) \neq D_{\beta}\left[\partial_{3} F\right]\left[y_{*} ; \zeta_{*}\right]_{\beta}(t)$ and $\int_{a}^{b} \partial_{6} F\left[y_{*} ; \zeta_{*}\right]_{\beta}(t) d_{\beta} t \neq 0$.

Let us suppose we are in case (i). If we restrict $\eta_{2}$ to those functions such that $\eta_{2}(a)=\eta_{2}(b)=0$, we get from (10) that

$$
\partial_{2} \psi(0,0)=\int_{a}^{b}\left(\partial_{2} F\left[y_{*} ; \zeta_{*}\right]_{\beta}(t)-D_{\beta}\left[\partial_{3} F\right]\left[y_{*} ; \zeta_{*}\right]_{\beta}(t)\right) \cdot \eta_{2}(\beta(t)) d_{\beta} t
$$

Since $\partial_{2} F\left[y_{*} ; \zeta_{*}\right]_{\beta}(t)-D_{\beta}\left[\partial_{3} F\right]\left[y_{*} ; \zeta_{*}\right]_{\beta}(t) \neq 0$, then we can choose $\eta_{2}$ such that $\partial_{2} \psi(0,0) \neq 0$. Since $\psi(0,0)=0$, then by the Implicit Function Theorem there exists a function $h$, defined in a neighborhood $V$ of 0 such that $h(0)=0$ and $\psi\left(\varepsilon_{1}, h\left(\varepsilon_{1}\right)\right)=0$ for any $\varepsilon_{1} \in V$. This means that there exists a family of pairs $(y, \zeta)$ where

$$
\left\{\begin{array}{l}
y=y_{*}+\varepsilon_{1} \eta_{1}+h\left(\varepsilon_{1}\right) \eta_{2} \\
\zeta=\zeta_{*}+\varepsilon_{1} \delta_{1}+h\left(\varepsilon_{1}\right) \delta_{2}
\end{array}\right.
$$

Stat., Optim. Inf. Comput. Vol. 6, March 2018 
that satisfies the isoperimetric constraint. Moreover, $(0,0)$ is an extremizer of $\phi$ subject to the constraint $\psi=0$ and $\nabla \psi(0,0) \neq(0,0)$. Therefore, there is a Lagrange multiplier $\lambda \in \mathbb{R}$ such that

$$
\nabla \phi(0,0)=\lambda \nabla \psi(0,0) .
$$

Restricting $\eta_{1}$ to those such that $\eta_{1}(a)=\eta_{1}(b)=0$ we conclude that

$$
\partial_{1} \phi(0,0)=\int_{a}^{b}\left(\partial_{2} L\left[y_{*} ; \zeta_{*}\right]_{\beta}(t)-D_{\beta}\left[\partial_{3} L\right]\left[y_{*} ; \zeta_{*}\right]_{\beta}(t)\right) \cdot \eta_{1}(\beta(t)) d_{\beta} t
$$

and

$$
\partial_{1} \psi(0,0)=\int_{a}^{b}\left(\partial_{2} F\left[y_{*} ; \zeta_{*}\right]_{\beta}(t)-D_{\beta}\left[\partial_{3} F\right]\left[y_{*} ; \zeta_{*}\right]_{\beta}(t)\right) \cdot \eta_{1}(\beta(t)) d_{\beta} t
$$

Therefore

$$
\int_{a}^{b}\left(\left(\partial_{2} L\left[y_{*} ; \zeta_{*}\right]_{\beta}(t)-D_{\beta}\left[\partial_{3} L\right]\left[y_{*} ; \zeta_{*}\right]_{\beta}(t)\right)-\lambda\left(\partial_{2} F\left[y_{*} ; \zeta_{*}\right]_{*}(t)-D_{\beta}\left[\partial_{3} F\right]\left[y_{*} ; \zeta_{*}\right]_{\beta}(t)\right)\right) \cdot \eta_{1}(\beta(t)) d_{\beta} t=0
$$

and Lemma 2 proves that

$$
\partial_{2} L\left[y_{*} ; \zeta_{*}\right]_{*}(t)-D_{\beta}\left[\partial_{3} L\right]\left[y_{*} ; \zeta_{*}\right]_{\beta}(t)=\lambda\left(\partial_{2} F\left[y_{*} ; \zeta_{*}\right](t)-D_{\beta}\left[\partial_{3} F\right]\left[y_{*} ; \zeta_{*}\right]_{\beta}(t)\right)
$$

for all $t \in[a, b]_{\beta}$. Then

$$
\partial_{2} H\left[y_{*} ; \zeta_{*}\right]_{\beta}(t)=D_{\beta}\left[\partial_{3} H\right]\left[y_{*} ; \zeta_{*}\right]_{\beta}(t)
$$

for $H=L-\lambda F$ and all $t \in[a, b]_{\beta}$, proving that $H$ satisfies the general quantum Euler-Lagrange equation.

Since, by hypothesis,

$$
\int_{a}^{b} \partial_{6} L\left[y_{*} ; \zeta_{*}\right]_{\beta}(t) d_{\beta} t=0 \quad \text { and } \quad \int_{a}^{b} \partial_{6} F\left[y_{*} ; \zeta_{*}\right]_{\beta}(t) d_{\beta} t=0
$$

then it is obvious that

$$
\int_{a}^{b} \partial_{6} H\left[y_{*} ; \zeta_{*}\right]_{\beta}(t) d_{\beta} t=0 .
$$

Let us now consider the case (ii). If in equality (10) we restrict $\eta_{2}$ to those such that $\eta_{2}(a)=\eta_{2}(b)=0$ we get

$$
\partial_{2} \psi(0,0)=\int_{a}^{b} \partial_{6} F\left[y_{*} ; \zeta_{*}\right]_{\beta} \cdot \delta_{2} d_{\beta} t
$$

Since

$$
\int_{a}^{b} \partial_{6} F\left[y_{*} ; \zeta_{*}\right]_{\beta}(t) d_{\beta} t \neq 0
$$

we can choose $\delta_{2}$ such that $\partial_{2} \psi(0,0) \neq 0$. Applying the Implicit Function Theorem and the Lagrange multiplier rule, is possible to prove that there exists a real constant $\lambda$ such that for $H=L-\lambda F$, we have

$$
\int_{a}^{b} \partial_{6} H\left[y_{*} ; \zeta_{*}\right]_{\beta} d_{\beta} t=0
$$

Since,

$$
\partial_{2} F\left[y_{*} ; \zeta_{*}\right]_{\beta}(t)-D_{\beta}\left[\partial_{3} F\right]\left[y_{*} ; \zeta_{*}\right]_{\beta}(t)=0, \quad t \in[a, b]_{\beta}
$$

and

$$
\partial_{2} L\left[y_{*} ; \zeta_{*}\right]_{\beta}(t)-D_{\beta}\left[\partial_{3} L\right]\left[y_{*} ; \zeta_{*}\right]_{\beta}(t)=0, \quad t \in[a, b]_{\beta}
$$


it follows that

$$
\partial_{2} H\left[y_{*} ; \zeta_{*}\right]_{\beta}(t)-D_{\beta}\left[\partial_{3} H\right]\left[y_{*} ; \zeta_{*}\right]_{\beta}(t)=0, \quad t \in[a, b]_{\beta} .
$$

Finally, suppose we are in case (iii). The proof that $\left(y_{*}, \zeta_{*}\right)$ satisfies the Euler-Lagrange equation with respect to $H$ is similar to the case (i), if we consider in this case $\delta_{2}=0$. The proof that $\left(y_{*}, \zeta_{*}\right)$ satisfies the equality $\int_{a}^{b} \partial_{6} H[y ; \zeta]_{\beta}(t) d_{\beta} t=0$ is similar to the case (ii), considering here $\eta_{2}=0$.

Now we will prove the natural boundary conditions.

1. Suppose that $y(a)$ is free. If $y(b)$ is fixed, then $\eta_{1}(b)=0$; if $y(b)$ is free then we can restrict to all functions $\eta_{1} \in \mathcal{Y}$ such that $\eta_{1}(b)=0$. Therefore,

$$
\begin{aligned}
\partial_{1} \phi(0,0)= & \int_{a}^{b}\left(\partial_{2} L\left[y_{*} ; \zeta_{*}\right]_{\beta}(t)-D_{\beta}\left[\partial_{3} L\right]\left[y_{*} ; \zeta_{*}\right]_{\beta}(t)\right) \cdot \eta_{1}(\beta(t)) d_{\beta} t \\
& +\int_{a}^{b}\left(\partial_{4} L\left[y_{*} ; \zeta_{*}\right]_{\beta}(t) \cdot \eta_{1}(a)+\partial_{6} L\left[y_{*} ; \zeta_{*}\right]_{\beta} \cdot \delta_{2}\right) d_{\beta} t \\
& -\partial_{3} L\left[y_{*} ; \zeta_{*}\right]_{\beta}(a) \cdot \eta_{1}(a)
\end{aligned}
$$

and

$$
\begin{aligned}
\partial_{1} \psi(0,0)= & \int_{a}^{b}\left(\partial_{2} F\left[y_{*} ; \zeta_{*}\right]_{\beta}(t)-D_{\beta}\left[\partial_{3} F\right]\left[y_{*} ; \zeta_{*}\right]_{\beta}(t)\right) \cdot \eta_{1}(\beta(t)) d_{\beta} t \\
& +\int_{a}^{b}\left(\partial_{4} F\left[y_{*} ; \zeta_{*}\right]_{\beta}(t) \cdot \eta_{1}(a)+\partial_{6} F\left[y_{*} ; \zeta_{*}\right]_{\beta} \cdot \delta_{2}\right) d_{\beta} t \\
& -\partial_{3} F\left[y_{*} ; \zeta_{*}\right]_{\beta}(a) \cdot \eta_{1}(a) .
\end{aligned}
$$

Since $\nabla \phi(0,0)=\lambda \nabla \psi(0,0), \quad H$ satisfies the general quantum Euler-Lagrange equation and $\int_{a}^{b} \partial_{6} H\left[y_{*} ; \zeta_{*}\right]_{\beta}(t) d_{\beta} t=0$, then

$$
\partial_{3} H\left[y_{*} ; \zeta_{*}\right]_{\beta}(a) \cdot \eta_{1}(a)=\int_{a}^{b} \partial_{4} H\left[y_{*} ; \zeta_{*}\right]_{\beta}(t) \cdot \eta_{1}(a) d_{\beta} t .
$$

From the arbitrariness of $\eta_{1}$ it follows that

$$
\partial_{3} H\left[y_{*} ; \zeta_{*}\right]_{\beta}(a)=\int_{a}^{b} \partial_{4} H\left[y_{*} ; \zeta_{*}\right]_{\beta}(t) d_{\beta} t .
$$

2. Suppose now that $y(b)$ is free. Using similar arguments as the ones used in 1 . we prove that

$$
\partial_{3} H\left[y_{*} ; \zeta_{*}\right]_{\beta}(b)=-\int_{a}^{b} \partial_{5} H\left[y_{*} ; \zeta_{*}\right]_{\beta}(t) d_{\beta} t
$$

The proof is complete.

The previous result can be generalized for the case of abnormal extremizers to isoperimetric problems as follows.

Theorem 19 (Necessary optimality conditions for normal and abnormal extremizers to isoperimetric problems) Let us suppose that $L$ and $F$ satisfy hypotheses (H1)-(H3) and (i)-(iii) of Lemma 4. If $\left(y_{*}, \zeta_{*}\right)$ is an extremizer to the isoperimetric problem (8)-(9), then there exist two constants $\lambda_{0}, \lambda \in \mathbb{R}$, not both zero, such that $\left(y_{*}, \zeta_{*}\right)$ satifies the conditions

$$
\partial_{2} H[y ; \zeta]_{\beta}(t)=D_{\beta}\left[\partial_{3} H\right][y ; \zeta]_{\beta}(t), \quad t \in[a, b]_{\beta}
$$


and

$$
\int_{a}^{b} \partial_{6} H[y ; \zeta]_{\beta}(t) d_{\beta} t=0
$$

where $H=\lambda_{0} L-\lambda F$. Moreover,

1. if $y(a)$ is free, then

$$
\partial_{3} H\left[y_{*} ; \zeta_{*}\right]_{\beta}(a)=\int_{a}^{b} \partial_{4} H\left[y_{*} ; \zeta_{*}\right]_{\beta}(t) d_{\beta} t
$$

2. if $y(b)$ is free, then

$$
\partial_{3} H\left[y_{*} ; \zeta_{*}\right]_{\beta}(b)=-\int_{a}^{b} \partial_{5} H\left[y_{*} ; \zeta_{*}\right]_{\beta}(t) d_{\beta} t
$$

Proof

The proof is similar to the proof of Theorem 18, but in this case we apply the abnormal Lagrange multiplier rule. This result ensures the existence of two constants $\lambda_{0}$ and $\lambda$, not both zero, such that $\lambda_{0} \nabla \phi(0,0)=\lambda \nabla \psi(0,0)$. The rest of the proof is straightforward, so it will be omitted.

In the case where $L$ and $F$ do not depend on $y(a), y(b)$ and $\zeta$, and under appropriate assumptions on those functions $L$ and $F$, we obtain the following result for the simplest quantum isoperimetric problem.

\section{Corollary 4}

If $y_{*}$ is a local extremizer to problem

$$
\mathcal{L}[y]=\int_{a}^{b} L\left(t, y(\beta(t)), D_{\beta}[y](t)\right) d_{\beta} t \longrightarrow \text { extremize }
$$

subject to the integral constraint

$$
\mathcal{I}[y]=\int_{a}^{b} F\left(t, y(\beta(t)), D_{\beta}[y](t)\right) d_{\beta} t=\tau,
$$

then there exist two constants $\lambda_{0}, \lambda \in \mathbb{R}$, not both zero, such that $y_{*}$ satisfies the quantum Euler-Lagrange equation

$$
\partial_{2} H\left(t, y(\beta(t)), D_{\beta}[y](t)\right)=D_{\beta}\left[\partial_{3} H\right]\left(t, y(\beta(t)), D_{\beta}[y](t)\right),
$$

for all $t \in[a, b]_{\beta}$, where $H=\lambda_{0} L-\lambda F$. Moreover,

1. if $y(a)$ is free, then $\partial_{3} H\left(a, y(\beta(a)), D_{\beta}[y](a)\right)=0$;

2. if $y(b)$ is free, then $\partial_{3} H\left(b, y(\beta(b)), D_{\beta}[y](b)\right)=0$.

\section{Remark 6}

Again, if we restrict ourselves to the particular case where $\beta(t)=q t+\omega$, for some $q \in] 0,1[$ and $\omega \geqslant 0$, Theorem 29 and Theorem 30 generalize, respectively, Theorem 3.9 and Theorem 3.10 of [19] for the Hahn calculus of variations.

\section{Illustrative examples}

In this section we present some examples in order to illustrate our results. Although, to the best of our knowledge, a general result guarantying the existence of solutions of nonlinear second order $\beta$-difference equations, as well as a method to construct these solutions, have not yet been presented, we show here one example where it is possible to exhibit one solution to this kind of difference equations. For the particular case of second order linear homogeneous $\beta$-difference equations with constant coefficients, we refer the reader to [11] for the general solution. 


\section{Example 1}

Consider $\beta(t)=\ln t+1$, where $I=[1,+\infty[$. We remark that $\beta$ is a strictly increasing and continuous function that has a unique fixed point that is $s_{0}=1$. Moreover, $\beta$ satisfies inequality (1) where the equality holds only if $t=s_{0}$. Note also that $\beta(t) \leqslant t$ for all $t \in I$. Consider the following problem

$$
\left.\mathcal{L}[y]=\int_{1}^{3}\left(D_{\beta}[y]\right)^{2}-1\right)^{2} \cdot(y(\beta(t)))^{2} d_{\beta} t \longrightarrow \text { minimize }
$$

over all $y \in \mathcal{Y}$ satisfying the boundary conditions $y(1)=0$ and $y(3)=3$.

The quantum Euler-Lagrange equation (7) takes the form

$$
2 D_{\beta}\left[\left(\left(D_{\beta}[y](t)\right)^{2}-1\right) \cdot(y(\beta(t)))^{2} \cdot D_{\beta}[y](t)\right](t)=y(\beta(t)) \cdot\left(\left(D_{\beta}[y](t)\right)^{2}-1\right)^{2} .
$$

Consider

$$
y_{*}(t)=\left\{\begin{array}{ccc}
0 & \text { if } & t \in[1,2] \\
t & \text { if } & t \in] 2,+\infty[
\end{array}\right.
$$

Note that

$$
D_{\beta}\left[y_{*}\right](t)=\left\{\begin{array}{clc}
0 & \text { if } & t \in[1,2] \\
\frac{t}{t-\beta(t)} & \text { if } & t>2 \wedge \beta(t) \leqslant 2 \\
1 & \text { if } & t>2 \wedge \beta(t)>2
\end{array}\right.
$$

and therefore $y_{*}$ is an admissible function satisfying (12) (we remark that for all $t$ such that $t>2$ and $\beta(t) \leqslant 2$, $y(\beta(t))=0)$.

Noting that $s_{0}=1$, we can conclude by Theorem 5 that $\mathcal{L}[y] \geqslant 0$. Since $\mathcal{L}\left[y_{*}\right]=0$, it follows that $y_{*}$ is indeed a minimizer. Note that the minimizer $y_{*}$ is not continuous while in the classical calculus of variations we deal with functions that are necessarily continuous.

\section{Example 2}

Let $\beta: \mathbb{R} \rightarrow \mathbb{R}$ be such that $\beta(t)=q t+\omega$ for $q \in] 0,1[$ and $\omega \geqslant 0$. We remark that $\beta$ has a unique fixed point that is $s_{0}=\frac{\omega}{1-q}$. Consider the following problem

$$
\mathcal{L}[y, \zeta]=\int_{0}^{1} L[y ; t]_{\beta} d_{\beta} t \longrightarrow \text { minimize }
$$

where

$$
L[y ; t]_{\beta}=2 y(q t+\omega)+\left(D_{\beta}[y](t)\right)^{2}+(y(1)-2)^{2}+(1-\zeta)^{2}
$$

in the class of admissible functions $y \in \mathcal{Y}$ that satisfy the boundary condition $y(0)=0$. If $\left(y_{*}, \zeta_{*}\right)$ is a local minimizer to this problem, then, by Theorem $15,\left(y_{*}, \zeta_{*}\right)$ satisfies the following conditions:

$$
D_{\beta}\left[\partial_{3} L\right]\left[y_{*} ; \zeta_{*}\right]_{\beta}(t)=\partial_{2} L\left[y_{*} ; \zeta_{*}\right]_{\beta}(t) \Leftrightarrow D_{\beta}\left[D_{\beta}\left[y_{*}\right]\right](t)=1 ;
$$

(ii) $\quad \int_{0}^{1} \partial_{6} L\left[y_{*} ; \zeta_{*}\right]_{\beta}(t) d_{\beta} t=0 \Leftrightarrow \zeta_{*}=1$;

$$
\partial_{3} L\left[y_{*} ; \zeta_{*}\right]_{\beta}(1)=-\int_{0}^{1} \partial_{5} L\left[y_{*} ; \zeta_{*}\right]_{\beta}(t) d_{\beta} t \Leftrightarrow D_{\beta}\left[y_{*}\right](1)=2-y_{*}(1) .
$$

It is easy to check that

$$
y_{*}(t)=\frac{1}{q+1} t^{2}-\left(\frac{\omega}{q+1}-c\right) t+d
$$


where $c, d \in \mathbb{R}$, is a solution to the Euler-Lagrange equation given in $(i)$. Since $y_{*}(0)=0$, we conclude that $d=0$. Observing that

$$
D_{\beta}\left[y_{*}\right](t)=\frac{1}{q+1}(t+q t+\omega)-\left(\frac{\omega}{q+1}-c\right),
$$

then the natural boundary condition (iii) gives

$$
c=\frac{q+\omega}{2(q+1)}
$$

Hence, the pair $\left(y_{*}, 1\right)$ where

$$
y_{*}(t)=\frac{1}{q+1} t^{2}-\left(\frac{\omega}{q+1}-\frac{q+\omega}{2(q+1)}\right) t
$$

is a candidate to be a local minimizer for the problem. Moreover, since the Lagrangian function is jointly convex, Theorem 14 guarantees that $\left(y_{*}, 1\right)$ is a global minimizer.

\section{Example 3}

Suppose now that we want to minimize the following functional

$$
\mathcal{L}[y, \zeta]=\int_{0}^{1}\left(2 y(q t+\omega)+\left(D_{\beta}[y](t)\right)^{2}+\zeta y^{2}(0)+(y(1)-2)^{2}+(1-\zeta)^{2}\right) d_{\beta} t
$$

where $\beta(t)=q t+\omega$ for $q \in] 0,1[$ and $\omega \geqslant 0$, in the class of admissible functions $y \in \mathcal{Y}$ and $\zeta \in \mathbb{R}$.

If $\left(y_{*}, \zeta_{*}\right)$ is a local minimizer to this problem, then, by Theorem 15, we conclude that:

(i) $\quad D_{\beta}\left[\partial_{3} L\right]\left[y_{*} ; \zeta_{*}\right]_{\beta}(t)=\partial_{2} L\left[y_{*} ; \zeta_{*}\right]_{\beta}(t) \Leftrightarrow D_{\beta}\left[D_{\beta}\left[y_{*}\right]\right](t)=1 ;$

$$
\int_{0}^{1} \partial_{6} L\left[y_{*} ; \zeta_{*}\right]_{\beta}(t) d_{\beta} t=0
$$

$$
\partial_{3} L\left[y_{*} ; \zeta_{*}\right]_{\beta}(0)=\int_{0}^{1} \partial_{4} L\left[y_{*} ; \zeta_{*}\right]_{\beta}(t) d_{\beta} t
$$

(iv) $\partial_{3} L\left[y_{*} ; \zeta_{*}\right]_{\beta}(1)=-\int_{0}^{1} \partial_{5} L\left[y_{*} ; \zeta_{*}\right]_{\beta}(t) d_{\beta} t$.

Again, it is easy to check that

$$
y_{*}(t)=\frac{1}{q+1} t^{2}-\left(\frac{\omega}{q+1}-c\right) t+d
$$

where $c, d \in \mathbb{R}$, is a solution to the Euler-Lagrange equation given in $(i)$. In order to determine the values of $c, d, \zeta_{*}$ we will use condition $(i i)$ and the natural boundary conditions $(i i i)-(i v)$. Using condition $(i i)$ we conclude that

$$
\zeta_{*}=\frac{d^{2}+2}{2}
$$

The natural boundary condition $(i i i)$ allows us to conclude that $c=\frac{d^{3}+2 d}{2}$. Finally, using the natural boundary condition $(i v)$ we get that $d\left(d^{2}+3\right)=\frac{q+\omega}{q+1}$. Hence, the pair $\left(y_{*}, \frac{d^{2}+2}{2}\right)$, where

$$
y_{*}(t)=\frac{1}{q+1} t^{2}-\left(\frac{\omega}{q+1}-\frac{d^{3}+2 d}{2}\right) t+d \quad \text { and } \quad d\left(d^{2}+3\right)=\frac{q+\omega}{q+1},
$$

is a candidate to be a local minimizer for the problem. Note that if we restrict our problem for the case where the parameter $\zeta$ belongs to $\mathbb{R}^{+}$, then the Lagrangian function $L$ is jointly convex, and therefore, from Theorem 14, we can conclude that $\left(y_{*}, \frac{d^{2}+2}{2}\right)$ is a global minimizer. 


\section{Conclusion and perspectives}

In this paper we introduced the quantum variational calculus based on the general quantum differential operator $D_{\beta}$. We proved necessary optimality conditions of Euler-Lagrange type and natural boundary conditions for generalized problems of the calculus of variations where the Lagrangian function not only depend on time $t \in[a, b]$, function $y$ and the quantum derivative $D_{\beta}[y]$, but also on a real parameter $\zeta$ and the state values $y(a)$ and $y(b)$. We also provided a sufficient optimality condition.

There is still a lot of work to do in order to develop this new variational calculus. For example, one interesting direction is to extend our results to the higher-order case. In our opinion, the main difficulty is to prove the higherorder fundamental lemma of the calculus of variations because of the generality of the function $\beta$ (see Lemma 3.8 of [8] for the particular case of Hahn's variational calculus). Another open question is to consider problems of the general quantum variational calculus with time delay. Here, the main difficulty is that the chain rule, as known from classical calculus, does not hold for the general quantum derivative.

\section{Acknowledgments}

This work was supported by Portuguese funds through the Center for Research and Development in Mathematics and Applications (CIDMA) and the Portuguese Foundation for Science and Technology (FCT), within project UID/MAT/04106/2013.

\section{REFERENCES}

1. K. A. Aldwoah, Generalized time scales and associated difference equations, Ph.D. thesis, Cairo University, 2009.

2. K. A. Aldwoah, A. B. Malinowska and D. F. M. Torres, The power quantum calculus and variational problems, Dyn. Contin. Discrete Impuls. Syst. Ser. B Appl. Algorithms 19, 2012, no 1-2, 93-116

3. R. Almeida and D. F. M. Torres, Hölderian variational problems subject to integral constraints, Journal of Mathematical Analysis and Applications 359, 2009, Issue 2, 2009, 674-681.

4. R. Almeida and D. F. M. Torres, Nondifferentiable variational principles in terms of a quantum operator, Math. Methods Appl. Sci. 34, 2011, no. 18, 2231-2241.

5. R. Álvarez-Nordase, On characterizations of classical polinomials, J. Comput. Appl. Math. 196, 2016, 320-337.

6. G. Bangerezako, Variational q-calculus, J. Math. Anal. Appl. 289, 2004, 650-665.

7. A. M. C. Brito da Cruz and N. Martins, The $q$-symmetric variational calculus, Comput. Math. Appl., Volume 64, Issue 7, 2012, 2241-2250.

8. A. M. C. Brito da Cruz, N. Martins and D. F. M. Torres, Higher-order Hahn's quantum variational calculus, Nonlinear Anal. 75, 2012, no. 3, 1147-1157.

9. J. L. Cardoso and J. Petronilho, Variations around Jackson's quantum operator, Methods and Applications of Analysis 22 (4), 2015 , $343-358$.

10. J. Cresson, G. S. F. Frederico and D. F. M. Torres, Constants of motion for non-differentiable quantum variational problems, Topol. Methods Nonlinear Anal. 33, 2009, no. 2, 217-231.

11. N. Faried, E. M. Shehata and R. M. El Zafarani, On homogeneous second order linear general quantum difference equations, J. Inequal. Appl. 2017: 198.

12. M. Foupouagnini, Laguerre-Hahn Orthogonal Polynomials with respect to the Hahn Operator: Fourth-order Difference Equation for the rth Associated and the Laguerre-Freud Equations for the Recurrence Coefficients, Ph.D thesis, Universit é Nationale du B énin, B énin, 1998

13. W. Hahn, Über Orthogonalpolynome, die q-Differenzengleichungen genügen, Math. Nachr. 2, 1949, 4-34.

14. A. E. Hamza, Abdel-Shakoor M. Sarhan, Enas M. Shehata and Khaled A. Aldwoah, A general quantum difference calculus, Advances in Difference Equations, 2015:182.

15. K. A. Hoffman, Stability results for constrained calculus of variations problems: an analysis of the twisted elastic loop, Proceedings of the Royal Society A: Mathematical, Physical and Engineering Sciences, Volume 461, issue 2057, 2005, pp 1357-1381.

16. F. H. Jackson, $q$-Difference equations, Amer. J. Math. 32, 1910, no. 4, 305-314.

17. V. Kac and P. Cheung, Quantum calculus, Universitext, Springer, New York, 2002.

18. R. Koekoek, P. A. Lesky and R. F. Swarttouw, Hypergeometric orthogonal polynomials and their $q$-analogues, Springer Monographs in Mathematics, Springer, Berlin, 2010.

19. A. B. Malinowska and N. Martins, Generalized transversality conditions for the Hahn quantum variational calculus, Optimization, 2011, Vol. 62 , Iss. 3., 323-344.

20. A. B. Malinowska and D. F. M. Torres, The Hahn quantum variational calculus, J. Optim. Theory Appl. 147, 2010, no. 3, 419-442.

21. A. B. Malinowska and D. F. M. Torres, Quantum variational calculus, Springer Briefs in Electrical and Computer Engineering: Control, Automation and Robotics, Springer, New York, 2014. 
22. N. Martins and D. F. M. Torres, Higher-order infinite horizon variational problems in discrete quantum calculus, Comput. Math. Appl., Volume 64, Issue 7, 2012, 2166-2175.

23. J. Petronilho, Generic formulas for the values at the singular points of some special monic classical $H_{q, \omega}$-ortogonal polynomials, J. Comput. Appl. Math. 205, 2007, 314-324.

24. A. Zinober and S. Sufahani, A non-standard optimal control problem arising in an economics application, Pesqui. Oper., Volume 33, no.1, Rio de Janeiro, 2013. 